Revista Brasileira de Cartografia

ISSN 1808-0936 | https://doi.org/10.14393/revbrascartogr

Sociedade Brasileira de Cartografia, Geodésia, Fotogrametria e Sensoriamento Remoto

\title{
O Potencial do Sistema Nacional de Gestão de Informações Territoriais (Sinter) como Ferramenta de Transparência e Integração de Cadastros
}

\author{
The Sistema Nacional de Gestão de Informações Territoriais (Sinter) Potential as a Tool \\ for Transparency and Integration of Cadastres
}

Deborah Valandro de Souza ${ }^{1}$, Diogenes Cortijo Costa $^{2}$, Henrique Cândido de Oliveira ${ }^{3}$

${ }^{1}$ Instituto Federal do Espírito Santo - IFES, Coordenadoria de Estradas, Vitória, Brasil e Universidade Estadual de Campinas, Programa de Pós-Graduação em Engenharia Civil, Campinas, Brasil. E-mail deborah@ifes.edu.br.

ORCID: https://orcid.org/0000-0002-5508-4294

${ }^{2}$ Universidade Estadual de Campinas, Campinas, Brasil. E-mail: dcortijo@unicamp.br.

ORCID: https://orcid.org/0000-0003-0084-6252

${ }^{3}$ Universidade Estadual de Campinas, Campinas, Brasil. E-mail: hcandido@unicamp.br.

ORCID: https://orcid.org/0000-0002-2783-4668

Resumo: Este estudo busca analisar, a partir das informações já divulgadas sobre o Sistema Nacional de Gestão de Informações Territoriais (SINTER), criado em 2016 e administrado pela Receita Federal do Brasil, seu potencial como ferramenta de transparência e como sistema integrador de diferentes bases cadastrais. Metodologicamente o artigo adotou uma revisão bibliográfica exploratória e qualitativa, apresentando um histórico do Cadastro e do registro de imóveis no Brasil, passando por conceitos sobre o Land Administration Domain Model (LADM), modelo desenvolvido pela International Organization for Standardization (ISO), a criação do SINTER, do Cadastro Territorial Multifinalitário (CTM) como instrumento de gestão territorial, finalizando com uma abordagem acerca dos desafios que aguardam o SINTER após sua implementação, que residem principalmente, na realização de um CTM que atenda às diretrizes publicadas pela Portaria $\mathrm{n}^{\circ} 511$, do Ministério das Cidades.

Palavras-chave: Gestão Territorial. LADM. Cadastro Territorial Multifinalitário. Transparência Territorial.

\begin{abstract}
This study seeks to analyze, based on the information already disclosed about the Sistema Nacional de Gestão de Informações Territoriais (SINTER), created in 2016 and managed by the Receita Federal do Brasil, its potential as a tool for transparency and as an integrating system of different registration bases. Methodologically, the article adopted an exploratory and qualitative bibliographic review, presenting a history of the Cadastre and Land Registry in Brazil, passing through concepts about the Land Administration Domain Model (LADM), model developed by the International Organization for Standardization (ISO), the creation of SINTER, of the Multipurpose Territorial Cadastre as an instrument of territorial management and, ending with an approach to the challenges awaits SINTER after its implementation, which are mainly in the achievement of a CTM that meets published guidelines by Portaria 511 of the Ministério das Cidades.
\end{abstract}

Keywords: Land Administration. LADM. Multipurpose Cadastre. Land Transparency.

\section{INTRODUÇÃO}

O Brasil possui um histórico de problemas em seus registros de imóveis, principalmente em virtude da diversidade de informações em cartórios e cadastros de seus municípios. Poucos são os cadastros urbanos em que são realizadas medições diretas dos limites dos imóveis, fato que resulta em listagens com áreas desprovidas de dados de natureza métrica e geodésica confiável (BRANDÃO, 2003).

Apontado como uma das iniciativas de melhoria da gestão territorial e de integrar os variados cadastros e o registro no país, além de se tratar de um tema de importância social e interesse público, este artigo aborda o Sistema Nacional de Informações Territoriais (SINTER), instituído pelo Decreto nº 8.764 de 2016, sob a responsabilidade da Receita Federal do Brasil (RFB).

Como objetivo geral, pretende-se analisar a potencialidade do sistema e, caso seja totalmente implementado, o mesmo poderá se transformar em uma ferramenta de transparência e apoio à organização e 
integração da informação cadastral do território nacional. O objetivo específico, visa abordar o Land Administration Domain Model (LADM), modelo de referência adotado pela International Organization for Standardization (ISO), ISO/FDIS 19152 (2012), que propõe especificações para padronização de aspectos comuns dos sistemas de administração da terra e, em particular, dos seus elementos centrais cadastrais e sua possível conexão com o SINTER.

O SINTER visa incluir os registros de imóveis urbanos e rurais em um único sistema, com o intuito de integrar dados de registros públicos e fiscais. A publicação do manual que definirá os detalhes sobre a sua execução, bem como o Projeto de Lei 3876/15 apelidado de "Lei do Cadastro Urbano" são instrumentos em início de um processo para a elaboração desta base cadastral nacional única (OLIVEIRA; BRAGHIROLLI; SOARES, 2018).

Ao funcionar como uma plataforma integradora de cadastros, a promessa é que o sistema poderá promover, entre outros aspectos, uma melhoria da gestão urbana, combate mais eficaz à corrupção e fornecer maior segurança jurídica aos cidadãos. Os dados que alimentarão o SINTER serão fornecidos pelos municípios, no entanto, estes precisarão investir em tecnologia para o georreferenciamento, procedimento que ainda está sendo discutido pela Receita Federal.

Como percurso metodológico, optou-se por uma pesquisa bibliográfica, exploratória e qualitativa. A escolha do método se justifica pelo fato da revisão bibliográfica contribuir para uma ampliação e domínio de conhecimentos em determinada área, além de ter o propósito de construir e fundamentar os conceitos utilizados neste artigo.

A consulta bibliográfica foi realizada por meio de livros, sites oficiais de órgãos governamentais, legislação, Food and Agriculture Organization (FAO), International Federation of Surveyors (FIG), portais de periódicos e bases de dados on-line de artigos especializados, teses e outras referências científicas, além de anais de Congressos e Simpósios sobre a temática. Como este artigo aborda o SINTER é importante ressaltar que, por ainda estar em fase de implementação, o procedimento de coleta de dados buscou identificar informações atualizadas, muitas vezes encontradas apenas em fontes secundárias.

\section{O REGISTRO DE IMÓVEIS NO CONTEXTO HISTÓRICO}

A análise histórica dos cadastros sugere que estes sempre foram uma resposta técnica às preocupações da autoridade local. Somente com a Revolução Francesa, em 1789, um novo período se abriu para os registros cadastrais. Sob o reinado de Napoleão, 1804 a 1814, a criação de cadastros passou a se basear em uma pesquisa geral de todas as parcelas e sua representação em um mapa, de acordo com uma abordagem científica e padrões técnicos. O cadastro napoleônico é a principal inspiração para os atuais sistemas cadastrais (STEUDLER; RAJAFABIRD; WILLIAMSON, 2004).

No Brasil, para doutrinadores como Lima (2011) e Miranda (2010), o serviço notarial e de registros começou a se distanciar do direito português a partir da lei $n^{\circ}$ 601/1850, a Lei de Terras, que estabeleceu normas sobre as terras devolutas no Império e das possuídas por título de sesmaria sem preenchimento das condições legais.

A lei foi regulamentada por meio do decreto $\mathrm{n}^{\circ} 1.318$, de 1854, conhecido como "Registro do Vigário". É a primeira documentação da propriedade conhecida no Brasil e determinava que todo possuidor de terras, obrigatoriamente, deveria registrar suas propriedades na paróquia local. Nas declarações deveriam constar o local do imóvel, área, limites e confrontantes, se conhecidos. É interessante constatar que ainda hoje se utiliza este processo, no entanto de forma mais complexa.

A inscrição de todas as hipotecas legais se tornou obrigatória a partir da lei $\mathrm{n}^{\circ}$. 3.272/1855, originada do "Projeto de Lei Hipotecária". O projeto converteu-se na lei no 1.237 de 1864 e, conforme Fassa (2006), isso se deu devido ao Registro do Vigário não atender a todas as necessidades do Império. Em 1890, o decreto ${ }^{\circ}$ 370 incluiu entre os atos sujeitos a registro, a transmissão do domínio entre vivos. Com isso, foi estabelecido o registro imobiliário em instituição pública, de caráter jurídico, tornando-o apto a dar certeza à propriedade e garantia ao crédito real.

Em 1903, foi criado pela lei nº 973, o serviço público correspondente ao Primeiro Ofício Privativo e Vitalício do Registro Facultativo de Títulos, documentos e outros papéis, para autenticidade, conservação e 
perpetuidade dos mesmos e para os efeitos previstos no artigo $3^{\circ}$ da lei $\mathrm{n}^{\circ} 79$, de 1892 (regulamentada pelo decreto $n^{\circ}$ 4.775. Esse decreto, segundo Miranda (2010), inaugurou os cartórios no Brasil.

Uma evolução no Direito Notarial e de Registros pode ser percebida com a promulgação da lei $n^{\circ}$ 6.015 de 1973 e as alterações da lei $n^{\circ}$. 6.216. Ressalta-se a importância dessas leis na regulamentação das atividades relacionadas aos registros públicos.

Com as novas possibilidades técnicas surgiram os chamados cadastros de múltiplos propósitos ou multifinalitários. Nesse contexto, os registros de terras devem permitir a integração com base em uma planta topográfica, dos direitos de terra (despesas legais e obrigações relacionadas), a avaliação de terras e informações relativas ao uso da parcela ${ }^{1}$, integrando a gestão territorial com o planejamento, meio ambiente e desenvolvimento usando Sistemas de Informação Geográfica (SIG) (BRASIL,2010).

Em 1996, a International Federation of Surveyors ou Federação Internacional dos Geômetras (FIG), trouxe uma nova reflexão cadastral com a melhoria de sistemas cadastrais nos países em desenvolvimento. Isso resultou na Declaração de Bogor, que coloca o reconhecimento dos ativos imobiliários dos indivíduos no centro das preocupações de desenvolvimento socioeconômico.

Em 1999, segundo Carneiro (2003), a FIG apresentou uma declaração sobre cadastro e desenvolvimento sustentável que levou à formulação do conceito de propriedade, abrangendo as responsabilidades sociais e ambientais, bem como os direitos de aproveitar a propriedade. O cadastro, nesta perspectiva, está na encruzilhada de diferentes interesses e revela várias questões políticas, jurídicas e econômicas que alimentam a reflexão geográfica.

\section{O LAND ADMINISTRATION DOMAIN MODEL (LADM)}

Pesquisas realizadas nos últimos 15 anos procuraram desenvolver modelos conceituais de referência para os cadastros territoriais e padronizar termos e informações essenciais para sua operação e, dessa forma, contribuir com o aperfeiçoamento ou mesmo implantação de sistemas de administração de terras (FREDERICO; CARNEIRO, 2014).

O Land Administration Domain Model (LADM) é uma dessas padronizações. Desenvolvido pela International Organization for Standardization (ISO) e divulgado por meio da ISO:19152 - LADM, em 2012. O modelo, segundo Lemmen, Oosterom e Bennet (2015), é capaz de trazer efetividade aos sistemas cadastrais, desenvolvendo um esquema para atender a todos os tipos de posse, operar em ambientes formais e informais e ser adaptado para situações locais. Associado a Infraestrutura de Dados Espaciais (IDE), ressalta Lemmen (2012), o modelo poderá ser um instrumento para a implantação de políticas relacionadas com a posse, valor e uso da terra e às suas estratégias de gestão em prol do desenvolvimento economicamente e ambientalmente sustentável.

O LADM procura definir um modelo de referência que possa abranger os componentes básicos relacionados à administração de terras e elementos acima e abaixo da superfície. O modelo conceitual consiste em quatro grupos de componentes para modelagem do cadastro territorial com foco em Direitos, Restrições e Responsabilidades que afetam a terra, água e seus componentes geoespaciais: (a) As Partes (Parties)relacionando pessoas e organizações com a terra, (b) Unidade Administrativa Básica (Administrative) - que incluem direitos de propriedade, restrições e responsabilidades, (c) Unidade Espacial (Spatial Units) principalmente parcelas e o espaço legal de edifícios e redes de serviços públicos e (d) Levantamento e Representação (Surveying and Representation) - tais como procedimentos de levantamento e suas representações espaciais (geometria e topologia) (ISO, 2012).

O sistema oferece toda a terminologia necessária para compartilhar a descrição de diferentes procedimentos formais ou informais em várias jurisdições, além de fornecer uma base conceitual para a formação de perfis nacionais ou regionais e promove a combinação de informações de administração de terras de diferentes fontes (GOGOLOU; DIMOPOULOU, 2015).

Sua modelagem, explicam Frederico e Carneiro (2011), tem como um dos objetivos permitir que as

\footnotetext{
${ }^{1}$ De acordo com o Art. $2^{\circ}$ da Portaria ministerial no 511 (BRASIL,2009), a parcela cadastral é a menor unidade do cadastro, definida como uma parte contígua da superfície terrestre com regime jurídico único.
} 
partes envolvidas se comuniquem, com base no vocabulário comum implícito no esquema. O objetivo é padronizar diferentes modelos cadastrais, de forma a permitir integração e comunicação de todas as partes envolvidas que fazem uso das informações cadastrais e estimular o desenvolvimento de aplicações de software, acelerando a implantação de sistemas de administração de terras, apoiando, assim, o desenvolvimento sustentável.

Frederico e Carneiro (2011), entretanto, ressaltam a dificuldade na modelagem de um sistema LADM no Brasil, pela diversidade dos sistemas cadastrais envolvidos. Cadastros Rurais são mantidos pelo Instituto Nacional de Colonização e Reforma Agrária (INCRA) e pela Receita Federal, e os municípios mantém seu Cadastro Urbano. Além disso, existem cadastros ambientais em todas as esferas.

Apesar desta complexidade, Scarassatti et al. (2014) destacam vantagens no uso do LADM, considerando-o capaz de trazer efetividade aos sistemas de cadastro. O que cada país deve fazer é personalizar o esquema LADM às suas necessidades. A flexibilidade do modelo e sua terminologia a partir de um vocabulário comum, se aplicada ao SINTER, traz a possibilidade de uma comunicação entre os sistemas cadastrais e as partes interessadas, estabelecendo padrões de informação que poderão ser compartilhados entre todas as regiões do país.

Assim, para que ações como o decreto $\mathrm{n}^{\circ}$ 6.666, que instituiu a Infraestrutura Nacional de Dados Espaciais (INDE), e o SINTER passem a subsidiar a convergência de uma ordem cadastral nacional será necessário estabelecer um padrão entre os cadastros territoriais, com base nas diretrizes da portaria $n^{\circ}$ $511 / 2009$ e no modelo LADM.

\section{SISTEMA NACIONAL DE GESTÃO DE INFORMAÇÕES TERRITORIAIS (SINTER)}

Coordenado pela Receita Federal do Brasil, a promessa é de que o SINTER integrará as informações em um banco de dados espacial georreferenciado, de forma a abranger o fluxo dinâmico de dados jurídicos produzidos pelos serviços de registros públicos ao fluxo de dados fiscais, cadastrais e dos imóveis urbanos e rurais. O sistema deverá ainda melhorar o acesso da União, Estados e Municípios às informações, possibilitando soluções para elevar a eficiência da gestão pública e da regularização fundiária (BRASIL, 2018).

O SINTER se constituirá em segmentos de bancos de dados denominados camadas, para que entes públicos, notários e registradores possam interagir sobre o mesmo mapa, criando sinergias que não seriam possíveis com cada um tendo o seu cadastro isolado (BRASIL, 2017).

Entretanto, para atingir esses objetivos e produzir resultados confiáveis, faz-se necessário que o cadastro seja padronizado, ou seja, com caráter multifinalitário, abrangendo dados do Cadastro Territorial (CT), dados de Registro de Imóveis (RI) e dos cadastros temáticos, integrando cadastros sempre atualizados, refletindo a realidade territorial do país. Assim sendo, o ideal é que o setor de cadastro urbano seja mantido pelas prefeituras que deverão se adequar a esta necessidade técnica fazendo uso das ferramentas disponibilizadas pelo SINTER.

A variedade de registros no território nacional pode gerar um obstáculo para sua efetividade, pois cria na prática uma situação de confusão de conceitos cadastrais, além de provocar a propagação de sistemas ineficientes, incapazes de apresentar dados reais de seus respectivos territórios. Isso resulta em desperdício do dinheiro público utilizado para o cadastro que, dessa forma, não atenderá às necessidades das municipalidades, como dados sobre o crescimento desordenado, cobrança do Imposto Predial e Territorial Urbano (IPTU), projetos de urbanização e outras atividades que envolvem gestão pública e urbana.

Nos imóveis rurais, as dificuldades encontradas na aplicação dos métodos em desuso passam a ser encontradas nas áreas urbanas de forma amplificada. Por esse motivo é importante analisar com cuidado os métodos a serem aplicados nos levantamentos cadastrais brasileiros, sobretudo quando o SINTER estiver em pleno funcionamento (ARAÚJO et al., 2018).

Varella, Oliveira e Moesch (2017) ressaltam que o SINTER veio como uma ferramenta capaz de reduzir problemas como sobreposição de registros, facilitar a execução de dívidas fiscais ou entre particulares, fornecer informações para o mercado de crédito relativas às garantias constituídas. Além disso, contribuir para dificultar a ocorrência de fraudes como lavagem de dinheiro e "grilagem" de terras ao proporcionar uma maior 
transparência das informações fundiárias acessíveis a partir de consultas a informações geoespaciais e controle dos bens individuais.

\section{O CTM COMO FERRAMENTA DE GESTÃO TERRITORIAL}

Países onde os sistemas cadastrais são mais eficientes possuem legislação própria sobre cadastro, e órgãos ou instituições que tratam especificamente do cadastro, além de parcerias público/privadas para o aperfeiçoamento e atualização cadastral, diferente do que acontece no Brasil (CARNEIRO, 2003).

O direcionamento e o suporte proporcionado pelo Cadastro Territorial Multifinalitário são entendidos como medidas para elaborar um plano de ação para um território e facilitar o processo de tomada de decisão. Porém, sem organização de dados ou com informações desatualizadas e equivocadas ele estará fadado ao fracasso e prejudicará qualquer gestão.

Diante do reconhecimento da importância do CTM, o Governo Federal, por meio do Ministério das Cidades, publicou a Portaria n ${ }^{\circ} 511$ de 2009, que estabeleceu as diretrizes para o CTM, possibilitando a reunião de informações a partir de técnicas de mapeamento, principalmente de imóveis do perímetro urbano (BRASIL, 2009). Esse é o principal documento que rege o CTM nos municípios brasileiros. O documento, entretanto, tem caráter orientador e não compulsório. $\mathrm{O}$ texto foi elaborado de forma generalista e não detalha os procedimentos técnicos a serem adotados, para que possa se adequar à diversidade das realidades municipais.

No Art. 20 da referida Portaria é conceituado a multifinalidade e os elementos mínimos:

Art. 20 O caráter de multifinalidade do CTM é assegurado pela integração de informações de outros sistemas ao sistema básico comum, de conteúdo mínimo, que favoreça a atualização.

$\S 1^{\circ}$ Considera-se como conteúdo mínimo do CTM a caracterização geométrica da parcela, seu uso, identificador único, localização e proprietário, detentor do domínio útil ou possuidor; $\S 2^{\circ} \mathrm{O}$ identificador único da parcela é a chave de ligação com o CTM e não deve ser confundido com os identificadores específicos definidos nos cadastros temáticos; [...] (BRASIL, 2009).

Já o Art. $4^{\circ}, 5^{\circ}$ e $9^{\circ}$ da mesma norma, afirma que deve haver a ligação entre os dados do CTM, com as informações contidas no Registro de Imóveis (RI), instituindo o Sistema de Cadastro e Registro Territorial (SICART). A associação entre o SICART e os cadastros temáticos recebe o nome de Sistema de Informação Territorial (SIT). As informações contidas no CTM e RI devem ser devidamente coordenadas e conectadas por meio de trocas sistemáticas de dados (BRASIL, 2009).

O conceito de um CTM oferece uma base espacial de integração dos direitos reais da propriedade com o uso, o valor e a distribuição natural e cultural dos seus recursos. Estudos cadastrais que tratam de problemas inerentes ao planejamento, urbanização, meio ambiente e governança territorial ajudam a destacar os laços inseparáveis entre geografia e política. Do ponto de vista geográfico, o CTM é um instrumento de poder e serve para conhecer, controlar e gerenciar territórios. Assim, o CTM é apresentado como um sistema de informação sobre a terra e, não mais, somente como um conjunto de plantas.

O conceito cadastral é enriquecido pelo uso da teoria dos sistemas, e mais especificamente dos Sistemas de Informação Geográfica (SIG), bem como das possibilidades tecnológicas oferecidas pela Geomática, possibilitando a gestão territorial.

Segundo Blachut (1974) o CTM se mostra um sistema de registro dos componentes espaciais, da realidade localizada nas cidades, identificado basicamente pelas formas geométricas, características e informações gerenciadas em códigos, que são utilizadas como dados para o planejamento urbano. Sendo também essencial à formulação de políticas públicas com fins de planejamento territorial e controle social, assim como uma ferramenta que sistematiza a coleta e a organização de informação, voltado para a integração de áreas distintas da administração pública e diversas operações inerentes à governança territorial.

Paixão, Nichols e Carneiro (2012), identificaram problemas para implantação do CTM no país como escassez de dados espaciais, comprovação documental descritiva sobre a propriedade real, falta de uma legislação nacional voltada ao cadastro que seja orientativa e baseada em um manual técnico consolidado, e o 
não cumprimento das legislações vigentes se configuram como entraves para o desenvolvimento de cadastros territoriais. Oliani (2016) acrescenta ainda como dificuldade a necessidade de integração entre o Registro de Imóveis e o Cadastro Físico, devidos aos custos demandados tanto para os particulares quanto para as prefeituras.

Ao analisar a conformidade do LADM com a Portaria $n^{\circ} 511$, Santos (2012) verificou que sua adoção para o Brasil é tecnicamente possível. Os resultados indicaram a compatibilidade do LADM para o cadastro urbano brasileiro, incluindo geometria e topologia das parcelas e aspecto legal.

O modelo LADM representa o estado-da-arte na área de gestão territorial no mundo. Ele é uma possibilidade de modelo padronizado para ser implementado nos sistemas cadastrais municipais do Brasil e a ser utilizado para estruturação do módulo integrador de Cadastros Territoriais do SINTER.

As normas e especificações técnicas, como o LADM e a Portaria n ${ }^{\circ} 511$ são fundamentais para associar todos os elementos do CTM (Lemmen, et al., 2009 e 2011), além da necessidade de ser consolidado sobre uma base espacial confiável, precisa e no caso do território brasileiro referenciado ao Sistema Geodésico Brasileiro (SGB) (BRASIL, 2009).

\section{O SINTER COMO FERRAMENTA DE TRANSPARÊNCIA E GESTÃO TERRITORIAL}

O SINTER propõe integrar níveis gráficos de mapeamento georreferenciado (camadas ou layers) às informações cadastrais e geoespaciais de acordo com as parcelas cadastrais. Trata-se, assim, de um banco de dados de gestão nacional do território, caracterizando-se como uma plataforma de informações que pode ser acessada a qualquer momento pelas diferentes esferas do governo, além de poder criar suas próprias camadas de visualização.

O sistema permitirá, por exemplo, aos registradores de imóveis e ao poder público, por meio de um visualizador gráfico dos limites da parcela territorial, comparar as camadas de imagem espaciais das áreas registradas (propriedade) com as das não registradas (posse) e, ao poder judiciário, ampliar os meios de fazer cumprir suas decisões em âmbito nacional e agilizar os processos de execução, com identificação e localização instantânea de bens sujeitos à penhora e o bloqueio de bens (BRASIL, 2016).

Para o Banco Central do Brasil, será uma ferramenta de consulta unificada em âmbito nacional das informações estatísticas, conjunturais e estruturais relativas ao mercado mobiliário e imobiliário, bem como às garantias reais constituídas em operações de crédito (BRASIL, 2016).

Um desafio para que o SINTER opere será o funcionamento do Cadastro Nacional de Imóveis Rurais (CNIR), assim como dos CTM urbanos e, para isso, será necessária a implementação de um cadastro efetivo, principalmente o cadastro urbano que não possui norma ou lei instituída. Estados e municípios não são obrigados a colaborar com o sistema, devido à falta de previsão legal e a inexistência de subordinação dos registros públicos à Receita Federal.

A realidade do cadastro territorial no Brasil é um empecilho, pois há prefeituras nas quais o cadastro existe, em outras está desatualizado ou inexiste. Nos 5.570 municípios brasileiros, existe um grande número em dificuldades financeiras e que não dispõe de cadastros atualizados, devido à deficiência tecnológica e falta de capacitação técnica na área. Outro desafio é a documentação a ser produzida, sujeita às mudanças institucionais e tecnológicas que podem ser incorporadas de acordo com cada governo

Somadas a todas essas dificuldades, pode-se ainda acrescentar uma possível controvérsia entre a transparência prometida pelo SINTER e a proteção aos dados pessoais, considerando que o referido sistema integra a base de dados aberta do Poder Público. O SINTER vai recolher informações classificadas pelo artigo $5^{\circ}$ da Lei Geral de Proteção de Dados e, como direito fundamental devem ser tomadas todas as medidas para evitar seu uso indevido (VARELA; OLIVEIRA; MOESCH, 2017).

Toda essa situação destaca um problema de integração e harmonização do direito privado da propriedade individual e do direito público de ordenamento territorial regional e local. A questão ocupa uma posição central na reflexão cadastral destinada a integrar cartas cadastrais de diferentes escalas (por exemplo, municipais). Os processos terão de ser alimentados por um conhecimento geográfico completo, detalhado e diversificado e as contribuições de um versátil sistema de informação cadastral para promover essas trocas são 
muito significativas e imprescindíveis para a sua consolidação.

Para Carneiro (2003), embora seja um instrumento de poder público, o cadastro deve se tornar um símbolo da democratização da governança territorial, facilitando a acessibilidade da informação geográfica a toda a sociedade e participação pública.

\section{CONSIDERAÇÕES FINAIS}

A desigualdade na distribuição da terra aumenta o nível de pobreza de um país. O Brasil , pela sua extensão territorial e diversas formas de registros de terras que possui, precisa de um sistema cadastral e de uma plataforma integradora dotada de um banco de dados geoespaciais e informações territoriais, com potencial para se tornar uma ferramenta de gestão do desenvolvimento, transparência e apoio à organização do território nacional.

Apesar de ainda existirem dúvidas sobre a capacidade do SINTER de se estabelecer como essa ferramenta, indo além das questões fiscais, uma vez que sua administração está subordinada à Secretaria da Receita Federal e não a órgãos de planejamento, o sistema pode resultar em um gerenciamento de informações mais rápido, atualizado e transparente, auxiliando gestores a planejarem melhor e evitarem ações que não trazem benefícios à sociedade.

É primordial que o CTM, principalmente o urbano, constitua-se uma estrutura sólida para que se torne confiável a fim de atender ao SINTER. É necessário que se institua uma legislação compulsória e normas com diretrizes para o estabelecimento, operacionalização e manutenção do cadastro urbano, assim como já aconteceu com o CNIR. A ausência desse instrumento para sistemas de administração territorial significa restrições à segurança da posse, à justiça social e ao desenvolvimento econômico.

Apesar do decreto $n^{\circ} 8.767 / 2016$ ser uma obrigatoriedade apenas para órgãos públicos federais e administração pública direta e indireta vinculados à União, isso não implica o insucesso na implantação, operação e manutenção do sistema. Entretanto, para que se tenha êxito é fundamental a colaboração dos Estados, Municípios e cartórios de notas e de registros. O referido decreto, no art. $3 .^{\circ}$, admite a necessidade de parcerias e convênios a serem firmados entre eles.

Ainda há muitos desafios a serem enfrentados pelo sistema até sua definitiva implementação e apresentação de resultados como uma plataforma de transparência confiável e integradora de informações notariais e registrais de vários órgãos e entidades. O SINTER possui a base tecnológica e jurídica para se tornar uma plataforma efetiva que contribuirá com o processo de aprimoramento da realidade brasileira frente à sistematização do CTM, organização dos dados geoespaciais, regularização fundiária e planejamento da gestão territorial. No entanto, o sucesso dessa iniciativa dependerá da cooperação de todos os entes envolvidos.

\section{Agradecimentos}

À Universidade Estadual de Campinas (UNICAMP), curso de Pós-Graduação em Engenharia Civil.

Ao Instituto Federal do Espírito Santo (IFES), campus Vitória, e à Coordenadoria de Estradas pela licença concedida a primeira autora para cursar o doutorado.

\section{Contribuição dos Autores}

A autora D.V.S foi responsável pela concepção da ideia do artigo, pela conceptualização, pela redação da minuta inicial, além da revisão e edição. Os autores D.C.C e H.C.O auxiliaram na conceptualização, na revisão e supervisão do desenvolvimento do artigo. Todos os autores participaram da revisão crítica e comentários.

\section{Conflito de Interesse}

Os autores declaram que não há conflitos de interesse. 


\section{Referências}

BLACHUT, T. J. Cadastre as a basis of a general land inventory of the country. In: Cadastre: various functions, characteristics, techniques and the planning of a modern land records system. Canada: National Research Council, 1974.

BRANDÃO, A. C. O princípio da vizinhança geodésica no levantamento cadastral de parcelas territoriais. 128 f. Tese (Doutorado em Engenharia de Produção) - Curso de Pós-Graduação em Engenharia de Produção, Universidade Federal de Santa Catarina, Florianópolis, 2003.

BRASIL. Decreto n. 8.764, de 10 de maio de 2016. Institui o Sistema Nacional de Gestão de Informações Territoriais e regulamenta o disposto no art. 41 da Lei $n^{\circ} 11.977$, de 7 de julho de 2009. Diário Oficial da União. Brasília, DF, maio. 2016.

BRASIL. Portaria $n^{\circ} 511$, de 7 de dezembro de 2009. Diretrizes para a criação, instituição e atualização do Cadastro Territorial Multifinalitário (CTM) nos municípios brasileiros. Diário Oficial da União. Brasília, DF, dez. 2009. Seção 1.

BRASIL. Receita Federal. Manual do SINTER. Módulo I - Integração com Informações Notariais e Registrais. Comitê Temático Registral e Notarial do SINTER, 2017. Disponível em: $<$ http://receita.economia.gov.br/sinter/manuais-operacionais/arquivos-e-imagens/manual-operacionalsinter-modelo-de-dados-registrais-e-notariais-v1_0.pdf>. Acesso em: 4 jun. 2019.

CARNEIRO, A. F. Cadastro imobiliário e registro de imóveis. Porto Alegre: Instituto de Registro Imobiliário do Brasil, 2003.

FASSA. O. R. C. Registrador de imóveis e responsabilidade patrimonial. São Paulo: Editora Juarez de Oliveira, 2006.

FOOD AND AGRICULTURE ORGANIZATION OF THE UNITED NATIONS (FAO). The state of food and agriculture. FAO Agriculture Series, 28. 1995. Disponível em: <http://www.fao.org/docrep/017/v6800e/v6800e.pdf>. Acesso em: 2 jun. 2018.

FREDERICO, L. N. S.; CARNEIRO, A. F. T. Considerações sobre a aplicação do Land Administration Domain Model (LADM) na modelagem do cadastro territorial brasileiro. V Simpósio Brasileiro de Ciências Geodésicas e Tecnologias da Geoinformação, Anais... Recife, 2014, p. 30 - 36. Disponível em: <https://www3.ufpe.br/geodesia/images/simgeo/papers/97-586-1-PB.pdf>. Acesso em:7 abr. 2019.

GOGOLOU, C.; DIMIPOULOU, E. Land administration standardization for the integration of cultural heritage in land use policies. Land Use Policy, v. 49, 2015, p. 617 - 625. Disponível em: <https://www.sciencedirect.com/science/article/abs/pii/S0264837715000617?via\%3Dihub>. Acesso em 03 mar. 2019.

INTERNATIONAL FEDERATION OF SURVEYORS (FIG). Statement on the Cadastre. Report prepared for the International Federation of Surveyors. by Commission 7, Copenhagen: Cadastre and Land Management. 1995.

INTERNATIONAL ORGANIZATION FOR STANDARDIZATION (ISO). ISO 19152:2012 Geographic Information: Land Administration Domain Model (LADM). Disponível em: <https://www.iso.org/standard/51206.html>. Acesso em: 10 abr. 2019.

LEMMEN, C. A. Domain model for land administration. PhD Thesis. Netherlands Geodetic Commission. 234 f. Royal Netherlands Academy of Sciences, The Netherlands, 2012. DOI: http://www.itc.nl/library/papers_2012/phd/lemmen.pdf.

LEMMEN, C.: OOSTEROM, P. V.; BENNETT, R. The Land Administration Domain Model. Land Use Policy, v. 49, 2015p. 535-545, 2015. Disponível em: 
<https://www.sciencedirect.com/science/article/pii/S0264837715000174>. Acesso em 25 fev. 2019.

LIMA, L. A. A atividade notarial e registral e sua natureza jurídica. Âmbito Jurídico, São Paulo, ano XIV, n. 92, set. 2011. Disponível em: https://ambitojuridico.com.br/cadernos/direito-administrativo/a-atividadenotarial-e-registral-e-sua-natureza-juridica/. Acesso em 22 jan. 2019.

MIRANDA, M. A. A importância da atividade notarial e de registro no processo de desjudicialização das relações sociais. Âmbito Jurídico, São Paulo, ano XIII, n. 73, fev. 2010. Disponível em: https://ambitojuridico.com.br/cadernos/direito-processual-civil/a-importancia-da-atividade-notarial-e-deregistro-no-processo-de-desjudicializacao-das-relacoes-sociais/. Acesso em 19 jan. 2019.

OLIANI, L. O. Noções de Cadastro Territorial Multifinalitário - CTM. Curitiba: Crea-PR, Série de Cadernos Técnicos, 2016.

OLIVEIRA, F. H.; BRAGHIROLLI, G.; SOARES, M. C. Determinação de limites de parcelas territoriais. Congresso Cadastro Técnico Multifinalitário e Gestão Territorial - COBRAC 2018, Anais...Florianópolis: UFSC, 2018.

PAIXÃO, S. K. S.; NICHOLS, S.; CARNEIRO, A. F. T. Cadastro Territorial Multifinalitário: dados e problemas de implementação do convencional ao 3D e 4D. Boletim de Ciências Geodésicas, v. 18, n. 1, p. $\quad 3-21,2012$. Disponível em: <http://www.scielo.br/scielo.php?pid=S1982$21702012000100001 \&$ script=sci_abstract\&tlng=pt>. Acesso em 10 jan. 2019.

SCARASSATTI, D. F.; RAMIREZ, E. J.; COSTA, D. C.; TRABANCO, J. L. A. Cadastro multifinalitário e a estruturação de sistemas de informações territoriais. Congresso Cadastro Técnico Multifinalitário e Gestão Territorial - COBRAC, Anais...Florianópolis: UFSC, 2014.

STEUDLER, DRAJABIFARD, A.; WILLIAMSON, I. P. Evaluation of land administration systems. Land use policy, $\quad$ v. $21, \quad$ n. 4 , p. $371 \quad-\quad 380, \quad 2004$. Disponível em: https://www.sciencedirect.com/science/article/pii/S0264837704000079?via\%3Dihb. Acesso em 09 jan. 2019.

VARELLA, M. D.; OLIVEIRA, C. G.; MOESCH, F. Salto digital nas políticas públicas: oportunidades e desafios. Revista Brasileira de Políticas Públicas, v. 7, n. 3, dez., p. 561 - 584, Brasília: Uniceub, 2017. Disponível em: < https://www.publicacoesacademicas.uniceub.br/RBPP>. Acesso em 28 j.; an. 2019

\section{Biografia do autor principal}

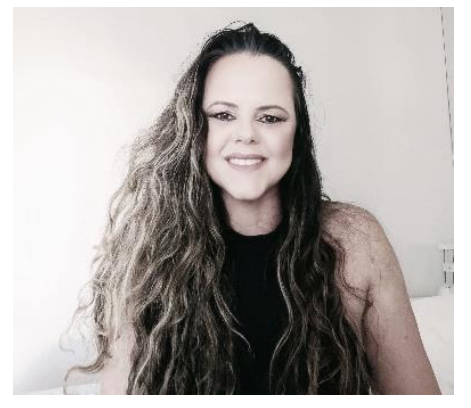

Deborah Valandro de Souza, Vitória-ES. Engenheira Agrimensora (2003) pela Universidade Federal de Viçosa. Mestre em Geofísica (2006) pelo Instituto de Astronomia, Geofísica e Ciências Atmosféricas (IAG) da Universidade de São Paulo. Em 07/2016 iniciou o doutorado em Engenharia Civil, na área de concentração em Transportes. Desde 2008 é docente do Instituto Federal do Espírito Santo, Campus Vitória, colaborando nos cursos de Estradas, Geoprocessamento, Engenharia Civil e Pós-Graduação Lato Sensu em Engenharia de Infraestrutura Urbana. Atua na área de Geomática, com ênfase em Geodésia, Cartografia e Cadastro aplicados ao Transportes, Gestão Territorial e Obras. 